\title{
Breast Milk: a Public Health Priority
}

\section{Giovannini M Barberi S Salvatici E Lops A Poli PC Riva E Banderali G}

Pediatric Department, San Paolo Hospital, University of Milan, Milan, Italy

\begin{abstract}
Substantial evidence documents the superiority of breastfeeding for mothers and breast milk for babies. Breast milk, with nutritional but also functional components, is a real biological system. According to present knowledge, it is associated not only with improved parameters of growth, but also to a better neuronalbehavioral development. Breastfeeding provides optimal nutrition for infants and is associated with decreased risk for infant and maternal morbidity and mortality. The importance of breastfeeding and the increasing evidence about the risks of not breastfeeding has put the support, promotion, and protection of breastfeeding at the forefront of many government and public health policies. The use of resources and the effort to promote and support breastfeeding should be the first investment for the health of children around the world. As maternity practices and hospital policies are known to influence breastfeeding, implement the Maternity Practices in Infant Nutrition and Care must be a public health priority.
\end{abstract}

Keywords: breastfeeding, breastfeeding support and promotion

\section{Breastfeeding: an ancient tradition}

The development of science and the economy depends on the introduction of technological innovations and new innovative methods. But the value of innovation for public health depends on its impact on promoting health.

Breastfeeding is not innovative, but although it has existed for 250-300 million years, its benefits have not yet surpassed by more innovative ways of infant feeding.

Innovation is about making changes, but when it comes to public health, it is not enough that innovation is something new: it is necessary to demonstrate that it is something useful.

\section{II . Breastfeeding as epigenetic factor}

Breast milk, with nutritional but also functional components, is a real biological system. According to present knowledge, it is associated not only with improved parameters of growth, but also to a better neuronal-behavioral development.

Breast milk is rich in antibodies and nutrients, and provides the child with a head start in health, growth and development. Breastfeeding also strengthens the bond between mother and child, which is good for the children's psychological development.

It is also associated with the prevention of some acute and chronic diseases. For example it is known the protective effect of breastfeeding against obesity and type 2 diabetes. A systematic meta-analysis showed that breastfeeding may reduce the risk of obesity in schoolchildren of $16-18 \%$, and the risk of type 2 diabetes of $11-55 \%$, compared with formula feeding (regardless of the biological and sociodemographic confounding variables) ${ }^{1}$. The relationship between nutrition in early life and genome may allow to understand the underlying mechanisms of disease that have high impact on individual health.

The risk of developing obesity depends on the interaction between genotype and individual lifestyles, but also environment and nutrition during fetal life and in the early ages of life are very important ${ }^{2}$. The epigenetic regulation of specific genes may become crucial in determining the individual risk for obesity.

Out of several variants identified in the PPAR $\gamma 2$ gene, the most common is the Pro12Ala substitution at codon 12. This polymorphism has been shown to be associated with reduced ability to transactivate responsive promoters and, in adults, with higher BMI, waist circumference, and obesity risk. In a recent study, the PPAR $\gamma$ Ala12 allele was associated with higher adiposity indexes (BMI, waist circumference and the sum of skinfolds) in adolescent who had not been breast-fed. However, this association was not seen in children who had been breast-fed (even for a short period). This result supports the hypothesis whereby breastfeeding has a beneficial effect on the obesity risk later in life in a genetically predisposed group. One potential hypothesis is that breast milk/breast-feeding supplies factors such as prostaglandin J2, a natural PPARG ligand. The decrease in PPARG2 transcriptional activity observed in Ala12 allele could be, therefore, compensated for by breast milk ${ }^{3}$.

The possibility that early infant feeding has long-term effects on blood cholesterol levels is supported by many studies. Breastfeeding seems to be associated with increased mean total cholesterol and LDL cholesterol levels in infancy but lower levels in adulthood/adult life. The high cholesterol content of breast milk may well be responsible. High cholesterol intake in infancy reduces endogenous synthesis of cholesterol, probably by down-regulation of hepatic hydroxymethyl glutaryl coenzyme A reductase ${ }^{4}$.

Many other positive and protective effects of human milk have been demonstrated in prevention of allergies, ear infections, infectious diseases and leukemia.

Breast milk is functionally positive not only for the child but also for the mother. Women with deleterious BRCA1 mutations who have breast-fed for a cumulative total of more than one year have a statistically significantly reduced risk of breast cancer.

\section{III . Breastfeeding: a Public Health Priority}

Considered the important role of human milk in the development of each one, support and promotion of breastfeeding should be a priority for each community.

There has been significant reliable evidence produced over recent years to show that breastfeeding is a major 
contributor to public health and has an important role to play in reducing health inequalities even in the industrialised countries of the world.

Structured breastfeeding programmes in maternity services shown to improve breastfeeding rates.

The importance of breastfeeding and the increasing evidence about the risks of not doing so has put the support, promotion, and protection of breastfeeding at the forefront of many government and public health policies.

Maternity practices in hospitals and birth centers throughout the intrapartum period, such as ensuring mothernewborn skin-to-skin contact, keeping mother and newborn together, and not giving supplemental feedings to breastfed newborns unless medically indicated, can influence breastfeeding behaviors during a period critical to successful establishment of lactation.

Maternity practices and hospital policies are known to influence breastfeeding therefore educating hospital staff to improve breastfeeding-related knowledge, attitudes, and skills; implementing a written hospital breastfeeding policy; and ensuring continuity of prenatal and postnatal breastfeeding education and support may improve newborn breastfeeding rates.

Incorporating care practices that include a number of the "Ten Steps to Successful Breastfeeding," as recommended by the Baby-Friendly Hospital Initiative, may increase the duration of exclusive breastfeeding after discharge.

The Baby Friendly Initiative, for example, is a worldwide programme of the World Health Organization and UNICEF.

It was established in 1992 to encourage maternity hospitals to implement the Ten Steps to Successful Breastfeeding and to practise in accordance with the International Code of Marketing of Breastmilk Substitutes.

To help in the implementation of this initiative, different tools and materials were developed, field-tested and provided, including a course for maternity staff, a self-appraisal tool and an external assessment tool. Since its launching BFHI has grown, with more than 152 countries around the world implementing the initiative. The initiative has measurable and proven impact, increasing the likelihood of babies being exclusively breastfed for the first six months.

To promote and support breastfeeding, WHO / UNICEF have developed and outlined a specific training. The course 20 hours is a concrete example of how to spread and standardize the preparation of the staff of the maternity wards about breastfeeding.

The aim of this course is that staff member will confidently support mothers with early and exclusive breastfeeding, and that this facility moves towards achieving Baby-friendly designation. Significant improvements in breastfeeding know-edge as a result of the 20-hour course occurred in all areas tested ${ }^{5}$.

In conclusion, the use of resources and the effort to promote and support breastfeeding should be the first investment for the health of children around the world.

The Centers for Disease Control and Prevention implements the Maternity Practices in Infant Nutrition and Care and requires governments that protection, promotion and support of breastfeeding are a public health priority.

\section{References}

[1] KA. Lillycrop, GC. Burdge, "Epigenetic changes in early life and future risk of obesity", Int J Obes, pp. 72-83, 2011.

[2] N. Liotto, M. Miozzo, ML. Giannì, F. Taroni, L. Morlacchi, P. Piemontese, P. Roggero, F. Mosca, "Early nutrition: the role of genetics and epigenetics", Pediatr Med Chir, pp. 65-71, 2009.

[3] RA. Waterland, KB. Michels, "Epigenetic epidemiology of the developmental origins hypothesis", Annu Rev Nutr, pp.363-88, 2007.

[4] CG. Owen, PH. Whincup, K. Odoki, JA. Gilg, DG. Cook, "Infant feeding and blood cholesterol: a study in adolescents and a systematic review", Pediatrics, pp. 597-608, 2002.

http:/www.who.int/nutrition/topics/bfhi/en/. 